\title{
Intra-Articular Proximal Phalanx Osteotomy Using a Three-Dimensional Printed Template: A Case Report
}

\author{
Yuhan Tan $^{a}$ Gino Vissers $^{b} \quad$ Bert Vanmierlo $^{a, c}$ Jean Goubau ${ }^{a, d}$ \\ aDepartment of Orthopaedics and Traumatology, University Hospital Brussels (Vrije \\ Universiteit Brussel - VUB), Brussels, Belgium; b Department of Plastic and Reconstructive \\ Surgery, University Hospital Antwerp, Edegem, Belgium; 'Upper Limb Unit, Department \\ of Orthopaedics and Traumatology, AZ Delta, Roeselare, Belgium; ${ }^{\mathrm{d} D e p a r t m e n t}$ of \\ Orthopaedics and Traumatology, AZ Maria Middelares Hospital, Ghent, Belgium
}

\author{
Keywords \\ Corrective osteotomy · Intra-articular fracture - Malunion · Phalangeal osteotomy · Three- \\ dimensional printing
}

\begin{abstract}
Malunion of an intra-articular fracture of the metacarpophalangeal joint can cause a dramatic effect, decreasing mobility and strength. For small joints, surgical options are often drastic; joint arthroplasty or arthrodesis. However, in cases of preserved joint space, an intra-articular osteotomy can be proposed. In order to improve preoperative planning, considering the small size of the articular surface and the importance of a precise osteotomy plane, we used a preoperative three-dimensional printed template to perform the intra-articular opening wedge osteotomy. This was followed by plate and screw osteosynthesis after adding a freeze-dried allograft to the created defect. The outcome at 1-year follow-up was more than satisfactory, with a normal range of motion and a restored, painless grip strength. A three-dimensional printed template is a useful, accurate, and efficient method to correct malunion of intra-articular fractures of the base of the proximal phalanx at the metacarpophalangeal joint.
\end{abstract}




\section{Case Reports in Orthopedic Research}

\section{Introduction}

Intra-articular fractures at the base of the proximal phalanx at the metacarpophalangeal joint often require open reduction and internal fixation. Unfortunately, in some cases, diagnosis is postponed due to inappropriate incidence of imaging or due to the slight morphological changes caused by the trauma at the articular surface, increasing the chance of missing the fracture. Moreover, these malunited fractures heal rapidly, making their possible surgical correction more complex. If left uncorrected, substantial disability may result from angular deformity, motional limitations and posttraumatic arthrosis [1].

Articular malunited step-offs need to be corrected and a stable construction should be created to allow early mobilization. Malunited intra-articular fractures at the base of the proximal phalanx can be treated by corrective osteotomy with or without concurrent tenocapsulolysis [2]. Success rates depend on a careful preoperative evaluation. Angular deformities need to be precisely measured in a three-dimensional way in order to plan the surgical procedure. Current technologies - such as three-dimensional printing - can be used to establish a more accurate preoperative evaluation. We report a case of a malunited intra-articular fracture at the base of the proximal phalanx treated by a corrective osteotomy using a threedimensional printed template.

\section{Case Report}

A 45-year-old female police officer was referred to our service by her insurance company after sustaining a hyperextension injury to her left ring finger in an attempt to arrest a suspect 18 months previously. She reportedly heard an obvious cracking, with her finger left in a hyperextended position, which she quickly "pulled back into the normal position" herself. She was initially treated at another center with a thermoplastic cast for 3 weeks. After removal of the splint, she complained of persistent loss of both range of motion (ROM) and strength. Options such as debridement, prosthesis, and arthrodesis were proposed to the patient. Due to the complexity of the injury, she was subsequently referred to our department of hand surgery.

Details of the clinical examination can be found in Table 1, showing both a decreased ROM and a loss of strength in the left ring finger. An initial radiograph and a cone beam computed tomography scan showed an intra-articular malunion of the dorsal aspect of the proximal phalanx, as shown in Figures 1 and 2.

A corrective osteotomy of the proximal phalanx was planned using a three-dimensional template. The cone beam computed tomography images were sent to a three-dimensional printing company (Materialise, Leuven, Belgium) and a patient-specific operative template based upon a three-dimensional printed model was created, as shown in Figure 3.

Surgery was performed using a dorsal approach to the metacarpophalangeal joint. The three-dimensional template was placed and temporarily fixated on the proximal phalanx. An opening wedge osteotomy and an angular articular correction were performed based upon the predetermined landmarks on the template, adding a supercrit ${ }^{\circledR}$ (LBTB - ISOS international Isos International, Munchbach, Luxemburg) allograft in the void created by the osteotomy. Figure 4 shows a reconstructed joint line, which was fixated using an intraoperatively bent plate and screw osteosynthesis. Immediate postoperative mobilization was allowed under supervision of a hand therapist.

At 1 year of follow-up, the patient was very satisfied with her pain reduction and functional results. The ROM of the left ring finger was almost symmetrical to the contralateral side. The clinical measurements at 1 year of follow-up are shown in Table 1.

\section{Karger'}


Table 1. Pre- and postoperative clinical measurement

\begin{tabular}{|c|c|c|c|c|}
\hline \multirow[t]{2}{*}{ Side (left/right) } & \multicolumn{2}{|c|}{ Preoperative } & \multicolumn{2}{|c|}{$\begin{array}{l}\text { Postoperative (1 year } \\
\text { follow-up) }\end{array}$} \\
\hline & left & right & left & right \\
\hline Metacarpophalangeal joint (MCP) ROM* & $+20 / 69$ & $+20 / 86$ & $+18 / 94$ & $+18 / 90$ \\
\hline Proximal interphalangeal joint (PIP) ROM* & $-30 / 95$ & $0 / 100$ & $-7 / 104$ & $+4 / 104$ \\
\hline Distal interphalangeal joint (DIP) ROM* & $0 / 60$ & $0 / 80$ & $+14 / 68$ & $+10 / 76$ \\
\hline Jamar strength, kg & 1 & 26 & 28 & 32 \\
\hline Jamar strength, kg & 0.5 & 2.5 & 2 & 2.5 \\
\hline Precision pinch strength, ${ }^{* *} \mathrm{~kg}$ & 0.5 & 2.5 & 2 & 3 \\
\hline DASH score ${ }^{* * *}$ & 106.8 & & 27.27 & \\
\hline
\end{tabular}

${ }^{*}$ Measured as degrees of extension/flexion.

** Measured as strength between the thumb and the fourth finger.

*** Disabilities of the Arm, Shoulder and Hand Score.

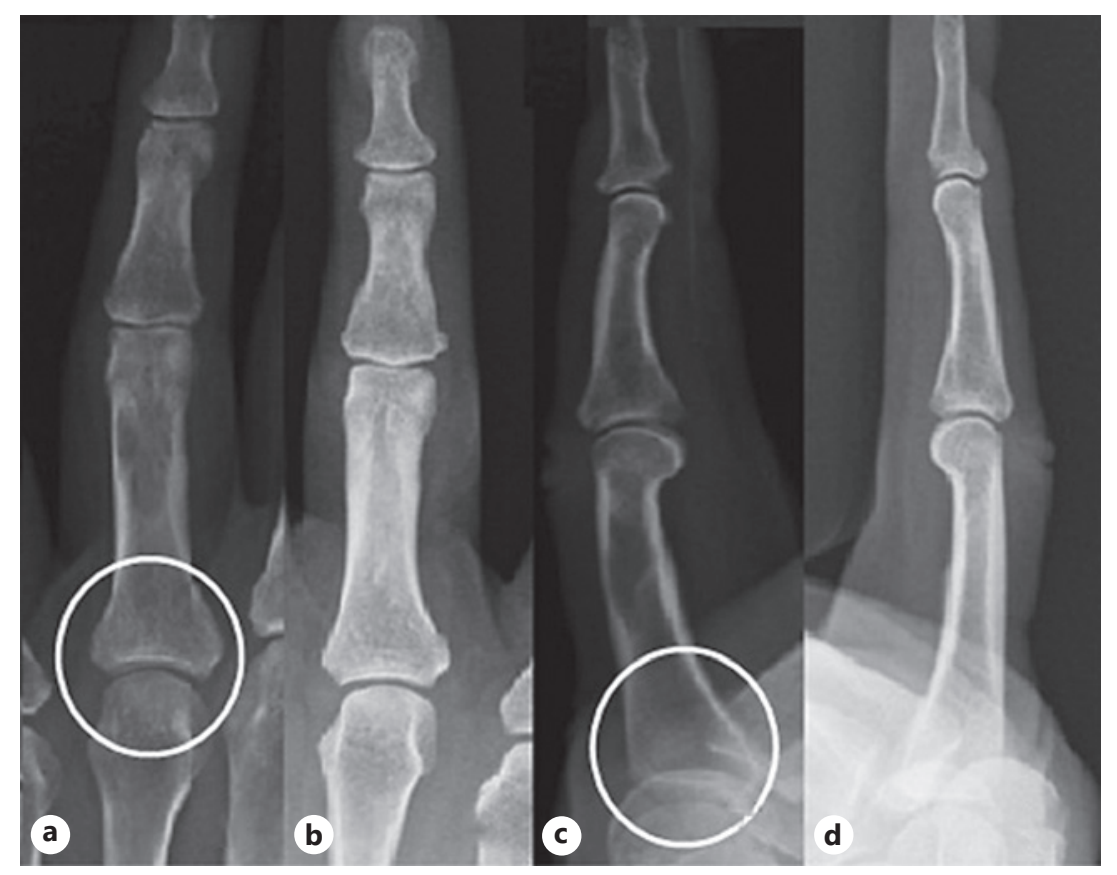

Fig. 1. a A/P radiograph of affected joint shows subtle double contour at the base of P1. b Contralateral unaffected A/P radiograph. c Sagittal plane radiograph of the affected joint shows an intra-articular step off due to impaction of the dorsal aspect of the joint. $\mathbf{d}$ Sagittal plane radiograph of the contralateral unaffected joint.

\section{Discussion}

Intra-articular malunions are known to pose both significant short- and long-term effects on joint function, especially in the younger population [3]. While treatment of extra-articular malunions of the phalanges has been largely successfully treated with osteotomies, the evidence on osteotomies for articular malunions of the proximal phalanges remains limited $[3,4]$. 
Case Reports in Orthopedic Research
Case Rep Orthop Res 2022;5:7-12

DOI: $10.1159 / 000521579$

(ब)

(c) 2022 The Author(s). Published by S. Karger AG, Basel www.karger.com/cio

Tan et al.: Phalangeal Osteotomy with 3D-Printed Template

Fig. 2. CBCT sagittal plane and $3 \mathrm{D}$ reconstruction shows an intra-articular step off at the P1 base. CBCT, cone beam computed tomography.
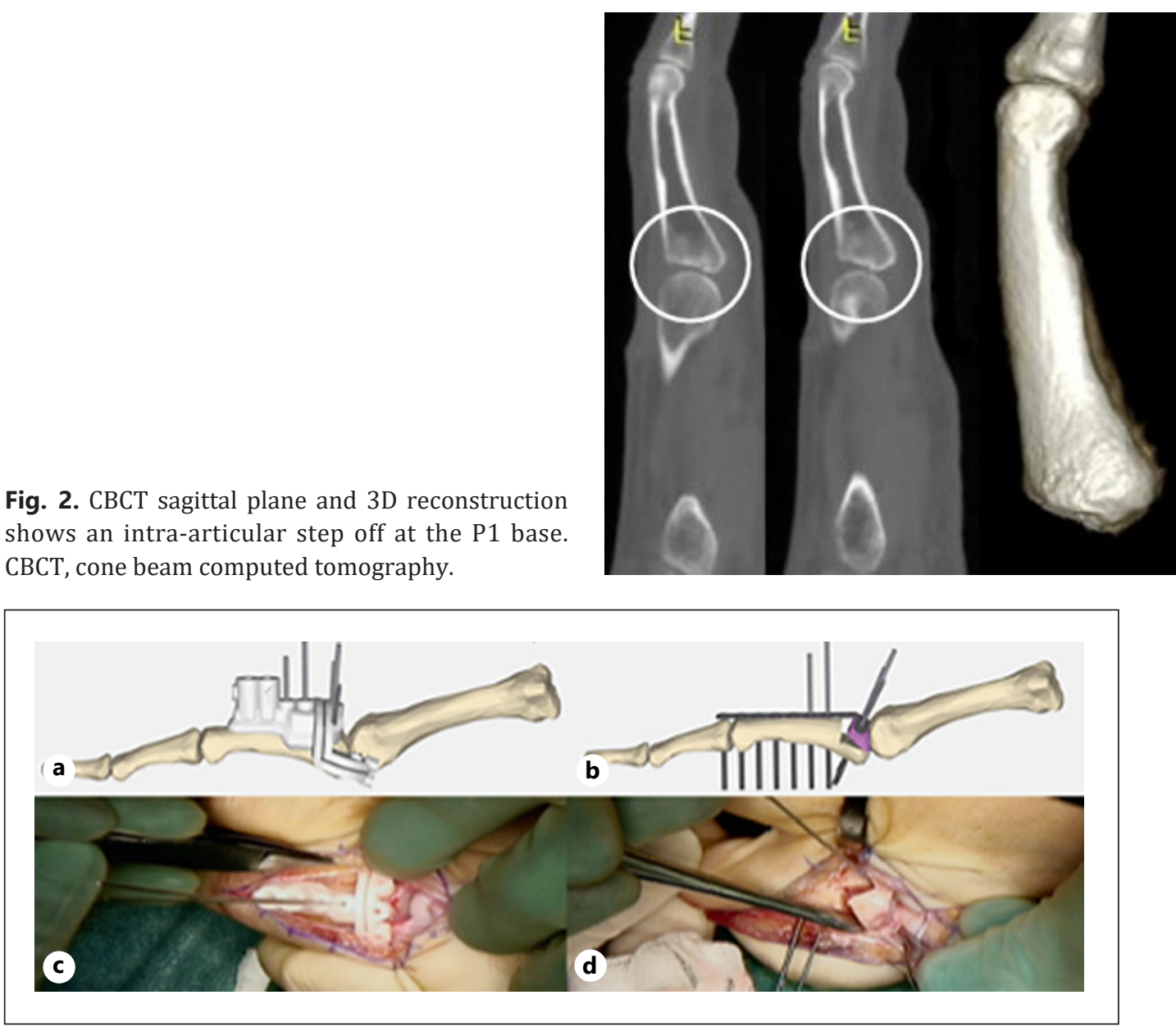

Fig. 3. a, b Preoperative planning. c Placement of three-dimensional template on the proximal phalanx with landmark of osteotomy site. d After execution of preoperative planned osteotomy sites.

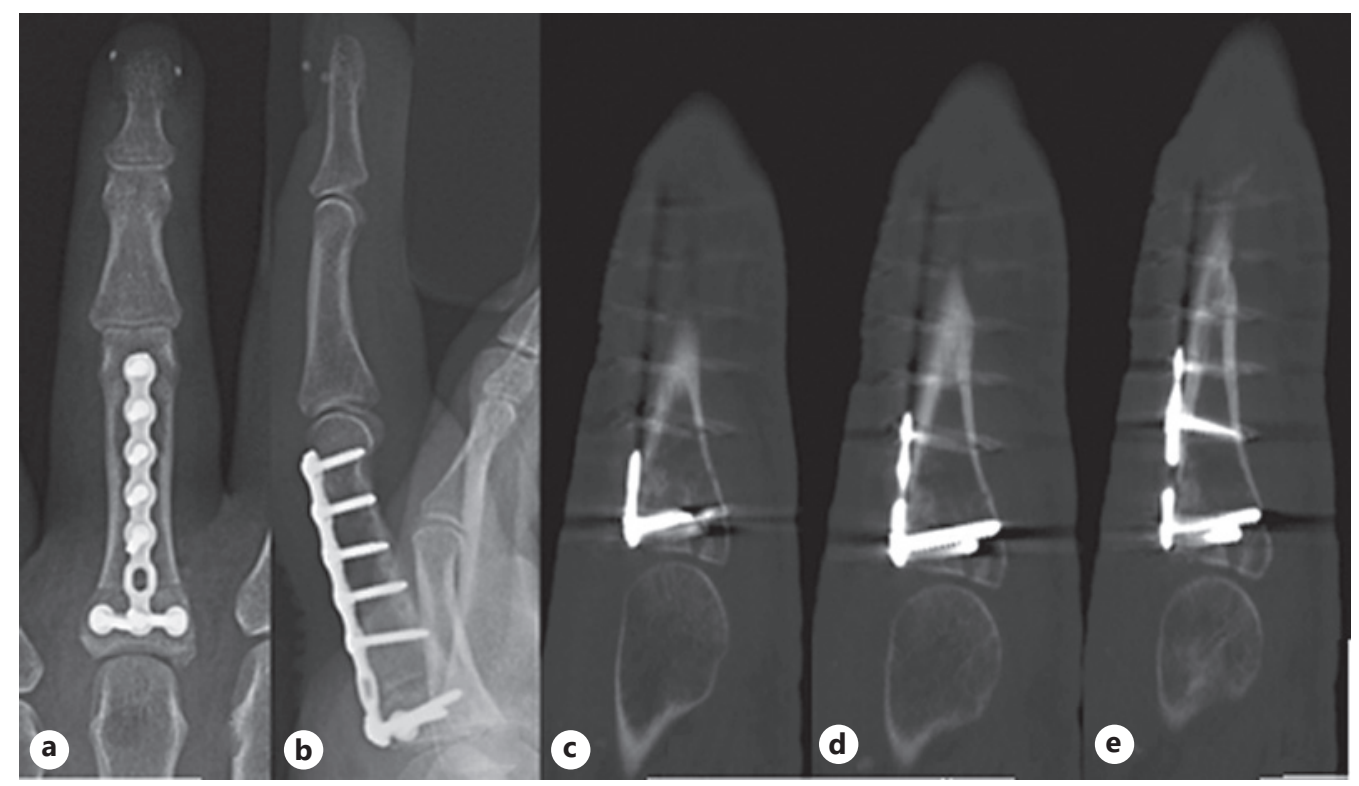

Fig. 4. Day 1 postoperative radiograph $(\mathbf{a}, \mathbf{b})$ and postoperative CBCT (c-e). Note the corrected articular surface. CBCT, cone beam computed tomography.

Karger' 
Extra-articular osteotomies for intra-articular fractures have been advocated in the past. This corrects angular and rotational deformities, but the joint surface remains incongruent. Persistent pain and limited joint motion are therefore often present [5-7]. Hirsiger et al. [8] reported satisfactory outcomes, correcting phalangeal and metacarpal malunions using 3D patient-specific templating, a similar technique to our case. However, their series only included one intra-articular malunion, which consisted of an metacarpal base intra-articular malunion [8].

Intra-articular osteotomies have shown to give better results in terms of alleviation of pain, correction of deformity, and improvement of ROM. However, current techniques have shown to be challenging and small fragment osteotomies have a potential risk of avascular necrosis $[9,10]$.

Careful preoperative planning of the osteotomy site is therefore essential in the treatment of these malunions. Preoperative three-dimensional and patient-specific template planning can provide a better understanding of the malunion and its correction, and can help to make the course of the procedure more predictable $[11,12]$. In our case, the preoperative threedimensional and patient-specific template planning gave us the possibility to execute an exact osteotomy with a minimum of biological harm in obtaining the correction.

Despite the fact that we had no previous experience using preoperative three-dimensional templating for phalanx malunion, we found the technique simple and straightforward. It resulted in good functional outcomes with a restoration of hand function. The most important disadvantage, however, is the higher cost (up to 2,500 EUR excl. VAT) and the time to produce the three-dimensional template. Although this surgical procedure evolved well in terms of survival of the fragments, these osteotomies remain at risk for potential avascular necrosis of the (small) mobilized fragment. We believe that this technique is a useful tool for treatment of intra-articular malunions, especially of the small bones. It allowed more precise visualization of the site of the deformity, as well as correct and predictable osteotomy cuts. The extra cost is offset by the permanent incapacity payment that insurance companies would have to make in the case of joint-sacrificing surgery.

\section{Statement of Ethics}

Full, written, well-informed approval for his case to be included in this study and for publication of images was given by the patient. No identifiers relating to the patient's identity are included in this article. Ethical approval was not required for this study in accordance with local guidelines.

\section{Conflict of Interest Statement}

The author(s) declared no potential conflicts of interest with respect to the research, authorship, and/or publication of this article.

\section{Funding Sources}

The author(s) received no financial support for the research, authorship, and/or publication of this article. 
Case Reports

in Orthopedic

Research
Case Rep Orthop Res 2022;5:7-12

DOI: $10.1159 / 000521579$

(๔) 2022 The Author(s). Published by S. Karger AG, Basel www.karger.com/cio

Tan et al.: Phalangeal Osteotomy with 3D-Printed Template

\section{Author Contributions}

Y. Tan contributed to the writing and editing of this article. J. Goubau contributed the case and contributed to editing this article. B. Vanmierlo and G. Vissers contributed to editing this article.

\section{Data Availability Statement}

All data generated or analyzed during this study are included in this article. Further inquiries can be directed to the corresponding author.

\section{References}

1 Light TR. Salvage of intraarticular malunions of the hand and wrist. The role of realignment osteotomy. Clin Orthop Relat Res. 1985;214:130-5.

2 Gupta A, Rut S. Corrective osteotomy for post-traumatic. J Hand Surg. 1996;21B(1):33-42.

3 Potenza V, De Luna V, Maglione P, Garro L, Farsetti P, Roberto C. Post-traumatic malunion of the proximal phalanx of the finger. Medium-term results in 24 cases treated by "In Situ" osteotomy. Open Orthop J. 2012; 6(1):468-72.

4 Freeland AE, Lindley SG. Malunions of the finger metacarpals and phalanges. Hand Clin. 2006;22(3):341-55.

5 Gollamudi S, Jones WA. Corrective osteotomy of malunited fractures of phalanges and metacarpals. J Hand Surg Br. 2000;25(5):439-41.

6 Bindra RR, Burke FD. Metacarpal osteotomy for correction of acquired phalangeal rotational deformity.J Hand Surg Am. 2009;34(10):1895-9.

7 Markiewitz AD. Complications of hand fractures and their prevention. Hand Clin. 2013;29(4):601-20.

8 Hirsiger S, Schweizer A, Miyake J, Nagy L, Fürnstahl P. Corrective osteotomies of phalangeal and metacarpal malunions using patient-specific guides: CT-based evaluation of the reduction accuracy. Hand. 2018;13(6): 627-36.

9 Teoh LC, Yong FC, Chong KC. Condylar advancement osteotomy for correcting condylar malunion of the finger. J Hand Surg Br. 2002;27(1):31-5.

10 Oak N, Lawton JN. Intra-articular fractures of the hand. Hand Clin. 2013;29(4):535-49.

11 Matter-Parrat V, Liverneaux P. 3D printing in hand surgery. Hand Surg Rehabil. 2019;38(6):338-47.

12 Belloti JC, Alves BVP, Faloppa F, Balbachevsky D, Netto NA, Tamaoki MJ. The malunion of distal radius fracture: corrective osteotomy through planning with prototyping in 3D printing. Injury. 2021;52 Suppl 3:S44-8. 